Introduction Parkinson's disease (PD) is a neuro-degenerative disorder with frequent involvement of the gut. Symptoms arise throughout the gastrointestinal tract through dysmotility secondary to autonomic and enteric nervous system involvement, as well from skeletal muscle involvement in the oropharynx and anorectum. It has been speculated that gut involvement may precede motor symptoms. The Wireless Motility Capsule (WMC) yields data on transit and motility throughout the gut. We report the first use of WMC to systematically assess motility in PD patients with and without gut symptoms, compared to controls. Methods 15 patients with established PD completed the study: eight ( $2 \mathrm{f}$, mean age 70 [47-85]) had GI symptoms and seven ( $2 \mathrm{f}$, mean age 61 [49-77]) did not based on history and baseline scores on the Gastroparesis Cardinal Symptom Index (GCSI) and Wexner constipation score. Data comparison with seven controls (3f, mean age 52 [39-63]). Medications affecting GI motility / $\mathrm{pH}$ were discontinued for the study and the WMC was ingested following a standardised nutrient bar meal. Data on gastric emptying time (GET), small bowel transit time (SBTT), colonic transit time (CTT) and whole gut transit time (WGTT) were calculated.

Results PD patients with gut symptoms showed significantly slower transit in the stomach (GET 5.2 vs. $2.7 \mathrm{~h}, \mathrm{p}=0.0003$ ), colon (CTT 57.8 vs. $27.4 \mathrm{~h}, \mathrm{p}=0.02$ ) and overall gut (WGTT 67.2 vs. $34.7 \mathrm{~h}, \mathrm{p}=0.02)$ compared to asymptomatic patients. Small Bowel transit (mean SBTT $4.17 \mathrm{~h}$ ) did not significantly differ. GET, SBTT, CTT and WGTT did not differ between asymptomatic PD and controls. There was a significant correlation between the Wexner constipation score and CTT in all patients $(\mathrm{p}<0.01)$, but no correlation between GCSI and gastric emptying $(\mathrm{p}>0.05)$.

Conclusion This study demonstrates that symptomatic PD patients have markedly delayed transit times throughout the whole gut compared to asymptomatic PD patients and controls. The correlation between scores and transit times suggest that WMC is a less useful indicator of gastric emptying than small bowel and colonic transit.

Disclosure of Interest None Declared.

\section{PWE-183 PRELIMINARY SIGNIFICANT FINDINGS FROM A RANDOMISED CONTROL TRIAL OF POSTERIOR TIBIAL NERVE STIMULATION IN SYSTEMIC SCLEROSIS ASSOCIATED FAECAL INCONTINENCE}

${ }^{1}$ SK Butt*, ${ }^{*} \mathrm{~A}$ Alam, ${ }^{1} \mathrm{~A}$ Raeburn, ${ }^{1} \mathrm{~J}$ Liwanag, ${ }^{2} \mathrm{VH}$ Ong, ${ }^{2} \mathrm{CP}$ Denton, ${ }^{3} \mathrm{CD}$ Murray, ${ }^{1} \mathrm{~N}$ Zarate-Lopez, ${ }^{1} \mathrm{~A}$ Emmanuel. 'Gastroenterology, UCL London, London, UK; ${ }^{2}$ Rheumatology, Royal Free Hospital, London, UK; ${ }^{3}$ Gastroenterology, Royal Free Hospital, London, UK

\subsection{6/gutjnl-2014-307263.443}

Introduction The gastrointestinal tract is affected in up to $90 \%$ of Systemic Sclerosis (SSc) patients with faecal incontinence (FI) being reported in up to $38 \%$. Passive faecal incontinence secondary to internal anal sphincter atrophy is the characteristic finding. We have shown that neuropathic changes are implicated in SSc patients with FI and sacral nerve stimulation has emerged as a potentially beneficial therapy in SSc. However this is expensive, invasive, not widely available and we have shown that medium term efficacy is poor. Posterior tibial nerve stimulation (PTNS) is a potential alternative to modulate the sacral plexus indirectly, with none of these disadvantages. This is the preliminary data on a randomised placebo controlled trial of PTNS versus sham PTNS to determine if nerve modulation is an effective treatment in SSc associated FI.
Methods We commenced a prospective randomised single-blind study of SSc patients with FI in February 2013 from a specialist Scleroderma unit. Baseline symptom scoring (bowel diary, Wexner), manometry and endoanal ultrasound were completed prior to randomization to PTNS or sham. PTNS was administered conventionally, by insertion of an acupuncture needle according to anatomical landmarks, connected to an electrical stimulator. Sham PTNS was administered in identical fashion but the PTNS surface electrode was not connected and instead separate TENS surface electrodes were connected to a TENS unit. Each patient underwent blinded intervention for 30 min periods, once a week for 12 weeks. The primary endpoints were the percentage reduction in faecal incontinence episodes and change in Wexner incontinence scores.

Results A total of 13 SSc patients (11 f), mean age 61 (36-72) completed the trial by October 2013. Of these 6 (5 f) underwent PTNS and 7 (6 f) patients underwent sham stimulation. All PTNS patients showed a reduction $(5-100 \%)$ in the number of FI episodes in comparison to 0 sham patients at 12 weeks ( $\mathrm{p}<$ 0.01 (CI: -81.49-14.34)). This matched an improvement in mean Wexner scores from baseline to treatment end (14.8 to 10.8 vs 13.4 to 13.6 , true vs sham respectively, $\mathrm{p}=0.03$.

Conclusion This pilot data is demonstrating significant effects of PTNS in Scleroderma-associated FI. We present this significant initial data but anticipate having at least 25 completed patients by May 2014.

Disclosure of Interest None Declared.

\section{PWE-184 THE EFFECT OF FIBRE ON CHRONIC CONSTIPATION IN ADULTS: A SYSTEMATIC REVIEW}

${ }^{1} S$ Christodoulides*, ${ }^{2}$ E Dimidi, ${ }^{3} \mathrm{KC}$ Fragkos, ${ }^{4} \mathrm{PG}$ McLean, ${ }^{1} \mathrm{Q}$ Aziz, ${ }^{2} \mathrm{~K}$ Whelan, ${ }^{1} \mathrm{SM}$ Scott. ${ }^{1}$ Neurogastroenterology Group, Queen Mary University of London, London, UK; ${ }^{2}$ Diabetes and Nutritional Sciences Division, King's College London, London, UK; ${ }^{3}$ Centre for Gastroenterology and Clinical Nutrition, University College London, London, UK; ${ }^{4}$ Nestlé Research Centre, Lausanne, Switzerland

\subsection{6/gutjn--2014-307263.444}

Introduction Symptoms of chronic constipation are common, with a prevalence of $\sim 14 \%$ in adults worldwide. Although increasing fibre intake is universally accepted as a first-line treatment, patient dissatisfaction is common. A systematic review is thus required in order to evaluate the quality of scientific evidence behind this management approach. The aim was to assess the effect of fibre on chronic constipation and IBS-C in adults via a systematic review of randomised controlled trials (RCTs). Methods Following Cochrane and PRISMA recommendations, references were identified by searching 5 electronic databases, hand-searching abstracts of 3 annual conferences, as well as scanning reference lists. Two reviewers independently assessed all studies identified, and performed data extraction and risk of bias assessment. Only RCTs reporting administration of fibre (carbohydrate polymers with $\geq 3$ monomers that are not hydrolysed by endogenous enzymes in the small intestine) in adults with either chronic constipation or IBS-C were included.

Results Of 916 records identified, only 9 RCTs were eligible (495 patients). Fibre supplements, administered in the form of powder, granules, yoghurt or milk, were: psyllium $(\mathrm{n}=3)$, galacto-oligosaccharides (GOS: $\mathrm{n}=2$ ), wheat bran $(\mathrm{n}=1)$, inulin $(\mathrm{n}=1)$, and mixtures of inulin/ resistant maltodextrin $(\mathrm{n}=1)$, and inulin/partially hydrolysed guar gum $(n=1)$. Outcomes measured included stool frequency $(\mathrm{n}=9)$, stool consistency ( $\mathrm{n}$ $=6)$, stool weight $(\mathrm{n}=5)$, faecal microbiota $(\mathrm{n}=3)$ and 
adverse events $(n=3)$. Four out of 9 RCTs demonstrated a significant increase in stool frequency following fibre consumption compared to placebo. Stool consistency significantly improved following fibre consumption in 2 RCTs, while 4 RCTs failed to show an effect. Three RCTs demonstrated a significant increase in stool weight, but 2 RCTs did not. Bifidobacteria significantly increased in 2 out of 3 RCTs (inulin and one GOS intervention). No side effects were observed and no patient withdrew because of adverse events. Attrition bias was high amongst trials, while selection bias and performance bias were unclear due to inadequate reporting. Outcome data will undergo meta-analysis.

Conclusion Current guidelines recommend the use of fibre as first-line treatment for constipation. However, this review shows that not all studies support its use in adults. Most studies suffer from small sample sizes and poor design with high risk of bias. The paucity of high quality data highlights the need for further large, methodologically rigorous RCTs.

Disclosure of Interest S. Christodoulides Grant/research support from: Nestec Ltd, E. Dimidi Grant/research support from: Nestec Ltd, K. Fragkos: None Declared, P. McLean Employee of: Nestec Ltd, Q. Aziz: None Declared, K. Whelan: None Declared, S. M. Scott: None Declared.

\section{PWE-185 BELIEFS ABOUT MANAGEMENT OF IRRITABLE BOWEL SYNDROME IN PRIMARY CARE: CROSS-SECTIONAL SURVEY}

UN Shivaji*, AC Ford. Leeds Gastroenterology Institute, St. James's University Hospital, Leeds, UK

\subsection{6/gutjnl-2014-307263.445}

Introduction There have been considerable advances in evidence synthesis concerning management of irritable bowel syndrome (IBS) in the last 5 years, with guidelines for its management in primary care published by the National Institute of Health and Care Excellence (NICE). We examined beliefs about IBS management among primary care physicians.

Methods This was a cross-sectional web-based questionnaire survey of 275 primary care physicians registered with three clinical commissioning groups in Leeds, UK. We e-mailed a link to a SurveyMonkey questionnaire, containing 18 items, to all eligible primary care physicians. Participants were given 1 month to respond, with a reminder sent out after 2 weeks.

Results One-hundred and two (37.1\%) primary care physicians responded. Eighty-four (82.4\%) of the respondents confirmed that they used clinical symptoms or signs elicited during the history and physical examination to diagnose IBS, with only 10 (9.8\%) using the Rome criteria, and 4 (3.9\%) the Manning criteria. A further 4 participants stated that they referred to a Gastroenterologist to confirm the diagnosis. Seventy (68.6\%) primary care physicians agreed or strongly agreed that IBS was a diagnosis of exclusion, with only 5 (4.9\%) strongly disagreeing with this statement. More than $80 \%$ checked coeliac serology often or always in suspected IBS. Between 56\% and 76\% believed soluble fibre, antispasmodics, peppermint oil, and psychological therapies were potentially efficacious therapies (table). The respondents were less convinced that antidepressants or probiotics were effective. Despite perceived efficacy of psychological therapies, $80 \%$ stated these were not easily available. Levels of use of soluble fibre, antispasmodics, and peppermint oil were in the range of $40 \%$ to $>50 \%$. Most primary care physicians obtained up-to-date evidence about IBS management from NICE guidelines.

Conclusion Most primary care physicians still believe IBS is a diagnosis of exclusion, and many are reluctant to use antidepressants or probiotics to treat IBS. More research studies addressing diagnosis and treatment of IBS based in primary are required.

Disclosure of Interest None Declared.

\section{PWE-186 UNDER-UTILISATION OF FAECAL CALPROTECTIN TO EXCLUDE IBD IN PATIENTS WITH FUNCTIONAL BOWEL DISORDERS}

VI Astle*, NR Lewis. Nottingham Digestive Diseases Centre, Nottingham University Hospital, Nottingham, UK

\subsection{6/gutjnl-2014-307263.446}

Introduction Calprotectin is a protein released by neutrophils in response to the presence of inflammation in the bowel ${ }^{1}$. Based on secondary care data with a cut-off of $50 \mathrm{mcg}$ using ELISA assay it has a sensitivity of $93 \%$ and specificity of $94 \%{ }^{2}$ to exclude inflammatory bowel disease (IBD) from functional bowel disorders. Calprotectin can be measured in a stool sample with this non-invasive test significantly cheaper than endoscopy and associated new patient referral ${ }^{3,4}$. Faecal calprotectin (FC) has also been shown to correlate with mucosal disease activity and can help to predict response to treatment or relapse in IBD ${ }^{1}$. We report the routine use of FC in gastroenterology practice at our hospital.

Methods All FC tests performed between 01/07/12 and 31/12/ 12 were systematically collected and reason for testing determined. Endoscopic, histological, radiological, laboratory and clinical records were systematically searched to identify tests performed in patients with FC results.

Results 294 FC tests were performed during the study period: $203(69.0 \%)$ for assessment of IBD and 91 (31.0\%) tests to exclude IBD in patients with suspected functional bowel disorders. Mean age of patients with suspected functional bowel disorders was 45 (SD 16.8) years and 62.6\% were female. Of the patients with suspected IBS who had a normal FC value $(\mathrm{n}=75), 50.7 \%$

\begin{tabular}{|c|c|c|c|c|c|}
\hline & Strongly agree & Agree & Neutral & Disagree & Strongly disagree \\
\hline Soluble fibre is an effective therapy (\%) & $3(2.9)$ & $54(52.9)$ & $39(38.2)$ & $6(5.9)$ & $0(0)$ \\
\hline Antispasmodics are an effective therapy (\%) & $6(5.9)$ & $71(69.6)$ & $25(24.5)$ & $0(0)$ & $0(0)$ \\
\hline Peppermint oil is an effective therapy (\%) & $4(3.9)$ & $64(62.7)$ & $30(29.4)$ & $3(2.9)$ & $1(1.0)$ \\
\hline Probiotics are an effective therapy (\%) & $1(1.0)$ & $20(19.6)$ & $56(54.9)$ & $22(21.6)$ & $2(2.0)$ \\
\hline TCADs are an effective therapy (\%) & $2(2.0)$ & $49(48.0)$ & $42(41.2)$ & $6(5.9)$ & $1(1.0)$ \\
\hline SSRIs are an effective therapy (\%) & $0(0)$ & $33(32.4)$ & $49(48.0)$ & $17(16.7)$ & $0(0)$ \\
\hline Psychological therapies are an effective therapy (\%) & $5(4.9)$ & $55(53.9)$ & 40 (39.2) & $1(1.0)$ & $0(0)$ \\
\hline
\end{tabular}

